\title{
Appropriate use of endoscopy in the diagnosis and treatment of gastrointestinal diseases: up-to-date indications for primary care providers
}

This article was published in the following Dove Press journal:

International Journal of General Medicine

29 October 2010

Number of times this article has been viewed

Vien X Nguyen'

Vi Thuy Le Nguyen ${ }^{2}$

Cuong C Nguyen'

'Department of Gastroenterology, Mayo Clinic, Scottsdale, AZ, USA;

${ }^{2}$ Department of Pharmacy, Banner Baywood Medical Center, Mesa,

AZ, USA
Correspondence: Vien X Nguyen Department of Gastroenterology, Mayo Clinic, 13400 East Shea Blvd, Scottsdale, AZ 85259, USA

$\mathrm{Tel}+\mid 4803016990$

Fax +I 4803016737

Email nguyen.vien@mayo.edu;

vnguy01@gmail.com
Abstract: The field of endoscopy has revolutionized the diagnosis and treatment of gastrointestinal (GI) diseases in recent years. Besides the 'traditional' endoscopic procedures (esophagogastroduodenoscopy, colonoscopy, flexible sigmoidoscopy, and endoscopic retrograde cholangiopancreatography), advances in imaging technology (endoscopic ultrasonography, wireless capsule endoscopy, and double balloon enteroscopy) have allowed GI specialists to detect and manage disorders throughout the digestive system. This article reviews various endoscopic procedures and provides up-to-date endoscopic indications based on the recommendations of American Society for Gastrointestinal Endoscopy and American Cancer Society for primary care providers in order to achieve high-quality and cost-effective care.

Keywords: endoscopy, endoscopic indications, endoscopic procedures, imaging, primary care, gastrointestinal disorders, appropriate use

\section{Introduction}

Open access endoscopy (OAE) is becoming more common due to the cost-driven health care system. It allows general practitioners to schedule elective common endoscopic procedures without prior specialty consultation. Endoscopies 'generally not indicated' are accounted for $49 \%$ when the examination is prescribed by general practitioners compared to $14 \%$ by endoscopists. ${ }^{1}$ Because of this disparity, it is critical for primary care physicians to have a thorough understanding of endoscopic indications. The purpose of this article is twofold: 1) to provide up-to-date endoscopic indications for primary care physicians so that only 'indicated' procedures are performed in order to avoid unnecessary complications and cost and 2) to familiarize primary practitioners with new endoscopic procedures in order to promote closed communication with gastrointestinal (GI) specialists (Table 1). The following information is based on the American Society for Gastrointestinal Endoscopy (ASGE) and American Cancer Society (ACS) recommendations.

\section{Definition of GI endoscopic procedures} Esophagogastroduodenoscopy

Esophagogastroduodenoscopy (EGD) is a procedure that visualizes the mucosal surfaces of the esophagus, stomach, and proximal duodenum. It allows both diagnostic and therapeutic functions. The gastroscope is used for EGD. It is a forward-viewing instrument and consists of a control head with valves for air insufflations and suction, an instrument channel for biopsy, an insertion length, and a bending length at the end of 
Table I A summary of appropriate indications for endoscopy

\begin{tabular}{|c|c|}
\hline Endoscopic procedures & Indications \\
\hline \multirow[t]{20}{*}{ EGD } & Achalasia \\
\hline & Barrett's esophagus \\
\hline & Caustic injury \\
\hline & Esophageal cancer \\
\hline & Esophageal strictures \\
\hline & Esophageal varices \\
\hline & Foreign bodies \\
\hline & Gastric epithelial polyps \\
\hline & Peptic ulcer disease \\
\hline & GERD \\
\hline & Gastric cancer \\
\hline & Ampullary adenoma \\
\hline & NDA \\
\hline & FAP/HNPCC \\
\hline & Inflammatory bowel disease \\
\hline & PEG \\
\hline & Upper Gl bleeding \\
\hline & Obscure GI bleeding \\
\hline & Pernicious anemia \\
\hline & Bariatric weight loss surgery \\
\hline \multirow[t]{12}{*}{$\mathrm{ERCP}$} & Choledocholithiasis \\
\hline & Benign biliary strictures/leaks \\
\hline & Pancreatic ductal leaks \\
\hline & Sphincter of Oddi dysfunction \\
\hline & Recurrent acute pancreatitis \\
\hline & Chronic pancreatitis \\
\hline & Pancreatic fluid collections \\
\hline & Bariatric weight loss surgery \\
\hline & IPMN \\
\hline & Ampullary adenoma \\
\hline & Cholangiocarcinoma \\
\hline & Pancreatic malignancy \\
\hline \multirow[t]{9}{*}{ EUS } & Esophageal/colorectal cancer \\
\hline & Gastric carcinoma/lymphoma \\
\hline & Mediastinal adenopathy \\
\hline & Pancreatic malignancy \\
\hline & Pancreatic cystic lesions \\
\hline & Submucosal tumors \\
\hline & Ampullary adenoma/NDA \\
\hline & Ampullary carcinoma \\
\hline & Inflammatory bowel disease \\
\hline WCE/DBE & Obscure GI bleed \\
\hline \multirow[t]{8}{*}{ Colonoscopy } & ACPO \\
\hline & Chronic diarrhea \\
\hline & Ampullary adenoma/NDA \\
\hline & Inflammatory bowel disease \\
\hline & Colorectal screening/surveillance \\
\hline & Colorectal cancer \\
\hline & Constipation \\
\hline & Lower Gl bleeding \\
\hline \multirow[t]{3}{*}{ Flexible sigmoidoscopy } & Colorectal screening \\
\hline & Young patients with colonic disease \\
\hline & Fulminant colitis \\
\hline
\end{tabular}

Abbreviations: EGD, esophagogastroduodenoscopy; ERCP, endoscopic retrograde cholangiopancreatography; EUS, endoscopic ultrasonography; WCE/DBE, wireless capsule endoscopy/double balloon enteroscopy; GERD, gastroesophageal reflux disease; NDA, nonampullary duodenal adenoma; FAP/HNPCC, familial adenomatous polyposis/hereditary nonpolyposis colorectal cancer; PEG, percutaneous endoscopic gastrostomy; GI, gastrointestinal; IPMN, intraductal papillary mucinous neoplasm; ACPO, acute colonic pseudo-obstruction. the scope. Patients should be kept nil per os (NPO) $6 \mathrm{~h}$ before the procedure. In preparation for EGD, the patient lies on the left side with the head resting on a pillow. A mouthguard is placed between the teeth to prevent biting on the endoscope. Then the gastroscope is inserted into the mouth, down to the esophagus, stomach, and ultimately ending in the second part of the duodenum. After this maneuver, the endoscope is withdrawn for further inspection of the GI lining. The fundus is examined by retroflexing the gastroscope's tip to resemble the J-shape. Any abnormal lesions should be biopsied. Most GI endoscopic procedures (EGD and colonoscopy) are performed under conscious sedation. The patient is able to respond to verbal or tactile stimulation. Combinations of benzodiazepines (midazolam and diazepam) and opioids (meperidine and fentanyl) are used for sedation. ${ }^{2}$

\section{Endoscopic retrograde cholangiopancreatography}

Endoscopic retrograde cholangiopancreatography (ERCP) is a technique that combines endoscopy and fluoroscopy to diagnose and to treat biliary or pancreatic ductal diseases. Similar to EGD, an endoscope is inserted through the mouth down into the duodenum. Instead of using a forward-viewing gastroscope as in EGD, a side-viewing duodenoscope is used for accurate identification of the ampulla of Vater, the opening of the common bile duct (CBD) and pancreatic duct (PD). When the ampulla is identified, a catheter is inserted through the ampulla into the CBD and/or PD. Radiocontrast dye is then injected into these ducts for real-time visualization by fluoroscopy. Fluoroscopy uses X-ray to look for strictures, blockages, or ductal leakages, and it guides therapeutic procedures. The bowel preparation is similar to EGD. However, because of longer duration, more sedatives than the standard benzodiazepines/opioid agents are needed for patient comfort. General anesthesia with propofol has demonstrated to be more effective than the above combinations in complex ERCP. ${ }^{2}$

\section{Endoscopic ultrasonography}

Endoscopic ultrasonography (EUS) uses a high-frequency ultrasound transducer that is incorporated into the tip of the endoscope to provide high-resolution images of the GI wall and adjacent structures. EUS-guided fine needle aspiration (EUS-FNA) is a technique using a thin needle under the ultrasonographic guidance to obtain an aspirate of the tissue. In addition, other therapeutic procedures, such as EUSguided drainage and EUS-guided celiac plexus blockage, can be performed under the guidance of real-time ultrasound. For upper EUS, bowel preparation is similar to EGD; for 
lower EUS, it is similar to colonoscopy (will be discussed under 'Colonoscopy'). More sedatives and analgesics may be required because these procedures are longer than EGD and colonoscopy. ${ }^{3}$

\section{Colonoscopy}

Colonoscopy allows for examination of the entire large bowel. The colonoscope is a forward-viewing instrument and has similar features to the gastroscope. As the patient lies on the left side, the endoscope is inserted through the anus, up the rectum, colon, and ultimately the terminal ileum. A thorough inspection is often done upon withdrawal of the colonoscope. Suspicious lesions are cauterized or removed for histopathology studies. Three accepted bowel preparations are polyethylene glycol (PEG), aqueous sodium phosphate $(\mathrm{NaP})$, and tablet NaP. With the glycol-based product, patients either drink 4 L of PEG (Colyte ${ }^{\circledR}$, GoLytely $\left.{ }^{\circledR}\right)$ in one dose the day before the procedure or in divided doses $(3 \mathrm{~L}$ the evening before and $1 \mathrm{~L}$ the morning of the procedure). The latter regimen is better tolerated. With the aqueous form, patients may take two doses of 30-45 mL of NaP (Fleet ${ }^{\circledR}$ ) (at least 10-12 h apart) along with plenty of fluids to prevent dehydration because $\mathrm{NaP}$ osmotically draws plasma water into the bowel lumen to promote cleansing. The tablet $\mathrm{NaP}$ $\left(\right.$ Visicol $\left.^{\circledR}\right)$ is in a pill form that has the same salt doses as found in the solution without the unpleasant taste. It is also formulated to limit the volume of liquid required. The total dose is 32-40 tablets ( 20 on the evening before the procedure and $12-20$ tablets $3-5 \mathrm{~h}$ before the colonoscopy). There are a few caveats with $\mathrm{NaP}$ laxative. It should be avoided in patients with congestive heart failure, renal impairment, and gut dysmotility due to significant fluid and electrolyte shifts. In addition, nonspecific aphthoid-like mucosal lesions have been associated with $\mathrm{NaP}$. If a diagnosis of inflammatory bowel disease (IBD) is being considered, NaP should be avoided. Similar to EGD, conscious sedation is used for patient comfort. ${ }^{4}$

\section{Wireless capsule endoscopy}

Until the introduction of wireless capsule endoscopy (WCE) in 1990s, diagnosing small bowel diseases was a challenge. Former techniques (Sonde enteroscopy and intraoperative enteroscopy) had many limitations and complications and were also associated with high mortality rate. WCE is purely a diagnostic tool. The system consists of a disposable 'video-recorder' capsule, a 7-h battery that allows images transmission to a data recorder that is carried around the patient's waist, and a computer workstation with proprietary software that reviews and interprets the results. WCE can be performed in an ambulatory outpatient setting. Without sedation, the capsule is ingested after a 10-12-h fasting period and moves through the small intestine by peristalsis. Although bowel preparation is not required, some centers advocate bowel catharsis for improved visualization of the small bowel. At present, studies yield conflicting results and no consensus has been reached.

\section{Double balloon enteroscopy}

While CE provides only a diagnostic workup, double balloon enteroscopy (DBE) allows for both diagnostic and therapeutic capabilities. DBE enables direct visualization of the small bowel by the push-and-pull technique with two balloons under fluoroscopic guidance. The system consists of a video endoscope, a flexible overtube, which covers the endoscope and enables it to slide freely, and two latex balloons that are inflated and deflated by a pressure-controlled pump system. The balloons are attached to the tip of the enteroscope and the overtube. The procedure is as follows:

1. The overtube balloon is inflated to 'grip' on the bowel lining.

2. The enteroscope is advanced as far as possible with its balloon deflated.

3. The enteroscope balloon is inflated to stabilize its position.

4. The overtube balloon is deflated and moved to the enteroscope balloon's tip.

The whole process (steps 1-4) is repeated.

DBE can be performed from the oral route, the anal route, or both. For the oral approach, the patient is kept NPO for at least $6 \mathrm{~h}$. For the anal approach, bowel cleansing is similar to colonoscopy. Total enteroscopy is not possible in a single procedure, and it is usually terminated when abnormalities are found and treated. Due to the lengthy procedure (70-80 min), general anesthesia is preferred. ${ }^{6}$ Serious complications of DBE are pancreatitis $(0.3 \%)^{7}$ and bowel perforation $(3.4 \%){ }^{8}$

\section{Flexible sigmoidoscopy}

Instead of examining the entire colon, flexible sigmoidoscopy (FS) only inspects the rectum, sigmoid, and descending colon. Although the sigmoidoscope is shorter, it shares many similar designs with the colonoscope. Since no sedation is required, the procedure can be performed in office-based settings by nurses or physician assistants. For bowel preparation, two Fleet enemas are adequate. Patients with active colitis or diarrhea do not need bowel preparation. After placing the 
patient in left lateral decubitus position, the scope is inserted into the rectum through the anus and is ascended until $40 \mathrm{~cm}$ is reached for a high-quality exam in colorectal cancer (CRC) screening. Polyps are biopsied and sent to pathology lab.

\section{Indications for EGD}

\section{Achalasia}

Achalasia is an esophageal motility disorder. In this condition, the smooth muscle of the esophagus has impaired peristalsis, and the lower esophageal sphincter (LES) fails to relax properly in response to swallowing. Some achalasia cases result from esophageal cancer or Chagas disease, a lower esophageal condition that is infected by a parasite, Trypanosoma cruzi. However, the cause for most achalasia is unknown. Symptoms include dysphagia, regurgitation of undigested food, coughing, and weight loss. Esophageal manometry is the 'gold standard' for diagnosing achalasia.' EGD is required to exclude esophageal cancer at the gastroesophageal junction before therapeutic interventions. The treatment goal is to reduce the LES pressure. This can be accomplished with pneumatic balloon dilators, botulinum toxin injection, or surgery as the last-line intervention strategy.

\section{Barrett's esophagus}

Barrett's esophagus is an acquired premalignant condition resulting from gastroesophageal reflux disease (GERD). It is marked by the presence of columnar epithelia in the lower esophagus, replacing the normal squamous epithelium. Compared to the normal population, patients with Barrett's esophagus have 30-125 times the risk of developing esophageal adenocarcinoma. ${ }^{10}$ However, screening and surveillance are currently still controversial. Currently, the ASGE makes the following recommendations: ${ }^{11}$

1. Screening for Barrett's esophagus: Screening EGD should be considered in selected patients with chronic, long-standing GERD ( $>5$ years). After a negative screening examination, further screening endoscopy is not necessary.

2. Barrett's esophagus with no dysplasia: For patients with established diagnosis of Barrett's esophagus, after two consecutive examinations with no dysplasia within 1 year, additional surveillance can be extended to 3 years.

3. Barrett's esophagus with low-grade dysplasia: For patients with low-grade dysplasia (LGD), an EGD should be performed at 6 months from LGD diagnosis with concentrated biopsies in the area of dysplasia. If LGD is confirmed, then annual surveillance endoscopy is recommended thereafter as long as dysplasia persists.

4. Barrett's esophagus with high-grade dysplasia: For patients with high-grade dysplasia (HGD), they can either elect to have treatment (photodynamic/thermal ablation or esophagectomy) or continue with surveillance. Patients who choose surveillance will have an EGD every 3 months for at least 1 year. After 1 year of no cancer detection and two consecutive endoscopies (at 3-month intervals) show no dysplastic changes, the surveillance interval can be extended.

5. Barrett's esophagus with indeterminate dysplasia: There may be a component of inflammation due to GERD. As a result, a repeat EGD should be performed after 8 weeks of acid-suppression therapy.

\section{Caustic injury}

Caustic injury is resulted from accidental or intentional ingestion of alkaline or acidic substances that include drain cleaners and other household cleaning products. Self-poisoning with these chemicals is common in Asia. The result is intense damage to the esophagus and stomach, often producing perforation. Late sequelae are stricture and squamous cell carcinoma. The incidence of carcinoma has been estimated to be $1 \%-4 \%$. After 12-20 years of injury, EGD is recommended. Subsequently, surveillance should not be more than every $1-3$ years. ${ }^{11}$

\section{Esophageal cancer}

Approximately 13,000 cases of esophageal cancer are diagnosed annually in the United States. Adenocarcinoma of the esophagus has one of the fastest rising incidence rates of any malignancy. EGD is the primary method for direct visualization and allows the endoscopist to obtain multiple biopsies. The classic endoscopic appearance is an ulcerated mass projecting from a rigid, aperistaltic segment of the esophagus. ${ }^{12}$ EGD also plays an important role in detecting recurrence and in treating esophageal cancer. ${ }^{13}$

\section{Esophageal strictures}

Sixty to seventy percent of all esophageal strictures are caused by GERD, and the remaining strictures are secondary to Schatzki's ring (a ring found in lower esophagus causing dysphagia), esophageal cancer, radiation therapy, esophageal surgery, eosinophilic esophagitis (a disorder characterized by infiltration of a large number of eosinophils in the esophagus), sclerotherapy, caustic injury, or photodynamic therapy. ${ }^{14}$ Dysphagia to solid foods is a common symptom. 
EGD with biopsies should be performed in all patients with dysphagia to exclude neoplastic processes. After ruling out malignancy, benign strictures are managed by either balloon or bougie dilations. ${ }^{9}$ Balloon dilators are often used to treat achalasia for forceful disruption of the LES. Bougies consist of two types: 1) mercury or tungsten-filled dilator and 2) wire-guided polyvinyl dilator. The former type has a tapered tip and can pass either blindly or under fluoroscopy. The latter type is passed over a guidewire and used for complex strictures. ${ }^{15}$

\section{Esophageal varices}

Variceal bleeding is a serious complication of portal hypertension with the mortality rate as high as $50 \% .{ }^{16}$ Because of high mortality, nonselective beta-blockers (propranolol or nadolol) have been shown to prevent or to delay the first episode of variceal bleeding. ${ }^{16}$ EGD is the only reliable method to confirm the presence of varices. Patients with cirrhosis and signs of portal hypertension (platelet count $<140,000 / \mathrm{mm}^{3}$ ) should have screening endoscopy. Endoscopic variceal ligation (EVL) is indicated for primary prophylaxis in patients who cannot tolerate beta-blockers (asthma). EVL has also become the treatment of choice for variceal hemorrhage.

\section{Foreign bodies}

Once foreign bodies are diagnosed, the timing of endoscopy is dependent on the risks of aspiration or perforation. Urgent endoscopic intervention is required to remove sharp objects or disk batteries that are lodged in the esophagus. Urgent EGD is also required to prevent aspiration when an object creates a high-grade obstruction. Conservative management is indicated when a foreign body has entered the stomach. If an object fails to pass the stomach within 3-4 weeks, it should be removed endoscopically. ${ }^{17}$

\section{Gastric epithelial polyps}

The majority of gastric epithelial polyps found during EGD are benign. However, adenomatous polyps have malignant potential. They should be completely resected. One-year surveillance endoscopy is reasonable to assess recurrence. If the result is negative, endoscopy should be repeated at 3-5-year intervals. ${ }^{11}$

\section{Peptic ulcer disease}

Dyspepsia is a condition characterized by a variety of symptoms (epigastric discomfort, bloating, anorexia, early satiety, belching, nausea, and heartburn). It can be caused by peptic ulcer disease (PUD), GERD, and malignancy. GERD and gastric cancer will be discussed in next two sections. Approximately, $15 \%$ of patients with dyspepsia have PUD, and the majority of them are tested positive for Helicobacter pylori antibody. ${ }^{18}$ In patients with PUD, H. pylori infection should be eradicated to improve healing and to reduce the risk of recurrence and rebleeding. At the experienced hands, $90 \%$ of gastroduodenal lesions can be detected by EGD. ${ }^{19}$ For patients with previous gastrectomy as a result of PUD, routine endoscopic surveillance is not indicated..$^{18}$

\section{GERD}

GERD is defined as symptoms or mucosal damage produced by the abnormal reflux of gastric contents into the esophagus. Patients with mild GERD symptoms (typical heartburn or mild intermittent dyspepsia) may be given an initial trial of therapy without endoscopic evaluation. EGD is the preferred procedure when patients with GERD have one of the following alarming symptoms: dysphagia, odynophagia, persistent or progressive heartburn symptoms while on antacid therapy, or weight loss. ${ }^{20}$ Sole hiatal hernia on the upper GI series is not an indication for EGD.

\section{Gastric cancer}

Gastric cancer is the fourth most common cancer in the world, only behind lung, breast, and colon cancers. However, it still remains the second most common cause of death from cancer worldwide. ${ }^{21}$ Multiple biopsies (at least seven specimens) of the gastric ulcers are necessary to rule out malignancy. It remains to be determined whether it is cost-effective to repeat endoscopy to verify post-treatment healing of gastric ulcers. At the present time, a follow-up endoscopy should be performed in 8-12 weeks after the initial EGD and treatment to document healing and repeat biopsies of any nonhealing ulcers. ${ }^{22}$

\section{Ampullary adenoma}

Adenoma arising at the major duodenal papilla is called ampullary adenoma. It can occur sporadically or in association with familial adenomatous polyposis (FAP) (will be discussed later). In order to determine the ampullary involvement, a side-viewing endoscope is used in addition to a routine forward-viewing duodenoscope. ERCP and EUS are important tools in selecting patients for endoscopic or surgical resection (will be discussed later). ${ }^{23}$ For patients undergoing endoscopic resection, postprocedure endoscopic surveillance should be done to ensure complete tissue removal and lack of disease recurrence. ${ }^{23}$ Endoscopic 
resection techniques are not standardized and should only be performed by experienced endoscopists.

\section{Nonampullary duodenal adenoma}

Nonampullary duodenal adenoma (NDA) occurs outside of the ampulla sporadically or in association with FAP (will be discussed in next section). Its endoscopic appearance is similar to Brunner's gland tumor, inflammatory polyp, carcinoid tumor, and hamartoma, thus a suspicious lesion should be biopsied before attempted resection. ${ }^{23}$ Before endoscopic resection, it is important to determine that the lesion does not involve the ampulla because the approach for evaluating an ampullary lesion is different (as noted above). Similar to ampullary adenomas, postprocedure endoscopic surveillance is indicated to verify complete resection and lack of occurrence. ${ }^{23}$

\section{FAP/hereditary nonpolyposis colorectal cancer}

The second leading cause of death in patients with FAP who have had colectomy is duodenal cancer. ${ }^{11}$ The risk of these patients developing duodenal adenocarcinoma is more than 100 -fold when compared to the general population. ${ }^{11}$ Both forward-viewing and side-viewing endoscopes should be used to detect adenomas in the duodenum, particularly in the periampullary region. Patients with hereditary nonpolyposis colorectal cancer (HNPCC) have a higher risk of developing cancers of the stomach and small bowel. Small bowel cancer in patients with HNPCC occurs at a young age (median age of 39 ), and the duodenum involves $50 \%$ of the cases. ${ }^{11}$ As a result, regular upper endoscopy surveillance of patients with FAP/HNPCC should be started around the time of colectomy or after the age of $30 .{ }^{11}$

\section{IBD}

Approximately $13 \%$ of patients with Crohn's disease (CD) have upper GI tract involvement. ${ }^{24}$ Duodenal biopsy often yields more granulomas (organized collections of macrophages are usually seen with CD) (40\%-68\%) than colonic biopsy. ${ }^{24}$ Hence, EGD is helpful in differentiating CD from ulcerative colitis (UC) in indeterminate colitis.

\section{Percutaneous endoscopic gastrostomy}

Percutaneous endoscopic gastrostomy (PEG) is an endoscopic procedure for placing a tube through the abdominal wall. Indications for PEG placement include impaired swallowing conditions (stroke), cancer of the oropharynx, larynx, and esophagus. PEG is also useful in patients with malignant bowel obstruction. PEG is not appropriate if peroral feeding is expected to resume within 30 days. Sometimes, the indication for PEG is debatable. In advanced dementia, PEG placement does not prolong life. Ethical considerations should be considered given the quality of life and prognosis. ${ }^{25}$

\section{Upper Gl bleeding}

Patients with upper GI bleeding (UGIB) should be stabilized before the initiation of endoscopy. Prompt endoscopy in patients with UGIB is effective in diagnosing and treating most causes of UGIB. It is also associated with reduction in both blood transfusion and length of hospital stay. ${ }^{26}$ Starting intravenous erythromycin 30-90 min before EGD promotes gastric motility and emptying and significantly improves the mucosal visibility. ${ }^{26}$

\section{Obscure GI bleeding}

Obscure GI bleeding is defined as persistence of bleeding from unknown origin, despite negative initial EGD and colonoscopy. A study demonstrated that push endoscopy diagnosed $64 \%$ of the bleeding lesions in patients with obscure GI bleeding within the reach of the standard endoscope. ${ }^{27}$ As a result, a 'second-opinion' EGD may be indicated in these patients before proceeding to WCE.

\section{Pernicious anemia}

Pernicious anemia is a form of megaloblastic anemia due to $\mathrm{B}_{12}$ deficiency in the setting of atrophic gastritis. Patients with this disorder have a 2-3-fold increase in the incidence of gastric cancer. Single endoscopy should be performed to identify prevalent lesions but routine subsequent endoscopic surveillance is not recommended. ${ }^{11}$

\section{Bariatric weight loss surgery}

Obesity is a major health problem that contributes to increased morbidity and mortality as well as to other disorders worldwide. In the United States, annual cost of managing obesity is approximately $\$ 100$ billion. ${ }^{28}$ Weight loss surgery is recommended for patients with the body mass index $(\mathrm{BMI}) \geq 40 \mathrm{~kg} / \mathrm{m}^{2}$ or those with $\mathrm{BMI} \geq 35 \mathrm{~kg} / \mathrm{m}^{2}$ and serious comorbid conditions. ${ }^{29}$ Patients with upper GI symptoms should be evaluated by EGD prior to undergoing bariatric surgery. A preoperative upper endoscopy is indicated for patients who are having gastric banding because a large hiatal hernia may be a contraindication. The European 
guidelines recommend preoperative upper endoscopy in all patients before surgery, regardless of whether or not symptoms are present. ${ }^{29}$ The rationale is that some bariatric surgical procedures render the stomach and duodenum inaccessible for upper endoscopic techniques. Nausea, vomiting, and abdominal pain are common symptoms commonly seen after bariatric surgery. However, patients with these persistent problems should have an upper endoscopy because the symptoms may indicate the presence of marginal ulcers, gastrogastric fistulas, postsurgical reflux disease, or anastomotic obstruction.

\section{Indications for ERCP Choledocholithiasis}

Choledocholithiasis is a disorder of gallstones in the CBD. Stone extraction ERCP is the treatment of choice for this condition with the success rate of more than $90 \% .{ }^{30}$ The overall complication rate and mortality rate are approximately $5 \%$ and less than $1 \%$, respectively. ${ }^{31}$

\section{Benign biliary strictures/leaks}

These benign conditions result from primary sclerosing cholangitis (PSC), congenital bile-duct abnormalities, chronic pancreatitis, or postoperative complications (liver transplantation). ${ }^{30}$ Endoscopic dilation is achieved by a graduated catheter, balloon, stent placement, or combination of interventions. Dominant strictures of patients with PSC should also undergo endoscopic brushing and biopsy for assessment of malignancy.

\section{Pancreatic ductal leaks}

Pancreatic ductal leaks occur as a result of acute pancreatitis, chronic pancreatitis, trauma, or surgical injury. Pancreatic leaks can lead to pancreatic ascites and pseudocyst formation. The leaks can be 'bridged' with transpapillary stents. ${ }^{30}$

\section{Sphincter of Oddi dysfunction}

Sphincter of Oddi dysfunction (SOD) is a condition of biliary colic resulting from the obstruction of bile and pancreatic juice flow. Patients with this disorder can have abnormal liver chemistries, dilated bile duct, or both. The gold standard to diagnose SOD is biliary manometry, which is performed during ERCP. ${ }^{30}$ Biliary sphincterotomy alleviate pain in the majority of patients. SOD manometry has an increased rate of pancreatitis and should be performed by experienced endoscopists.

\section{Recurrent acute pancreatitis}

If other imaging modalities of patients with recurrent acute pancreatitis are equivocal, ERCP should be done to evaluate the ductal anatomy along with bile analysis for microlithiasis. Endoscopic sphincterotomy without cholecystectomy is a viable option for patients with microlithiasis. ${ }^{30}$

\section{Chronic pancreatitis}

Both ERCP and EUS are useful for diagnosing CP, but ERCP should be reserved for patients in whom the diagnosis has not been established by less-invasive studies. ${ }^{32}$

\section{Pancreatic fluid collections}

ERCP can drain pancreatic fluid collections, such as acute pseudocyst, chronic pseudocysts, and pancreatic necrosis. Fluid collections communicating with the PD are drained via the transpapillary approach, while noncommunicating collections are amenable to the transmural (transgastric or transduodenal) therapy. The success rates for transpapillary and transmural routes are $>90 \%$ and $>80 \%$, respectively. ${ }^{30}$ Acute and chronic pseudocysts will be further discussed in the EUS section.

\section{Bariatric weight loss surgery}

Bariatric weight loss surgery has been discussed above in the EGD section. Postoperative rates for gallstones are high $(22 \%-71 \%)$, and cholecystectomy was performed in $7 \%-41 \%$ of patients who underwent bariatric surgery. ${ }^{29}$ The rates of choledocholithiasis in these patients are unknown. ERCP is challenging due to altered anatomy. According to a small study, ERCP with successful papilla cannulation was only achieved in $66 \%$ of patients. ${ }^{29}$

\section{Intraductal papillary mucinous neoplasm}

Intraductal papillary mucinous neoplasm (IPMN) is defined as papillary proliferations of pancreatic mucin-producing epithelial cells with/without excessive mucin hypersecretion and/or cystic ductal dilation. (Please refer to the EUS section for further discussion.) On ERCP, the pathognomonic for IPMN is the finding of mucus extruding from a patulous ampulla. ${ }^{33}$ Pancreatographic findings during ERCP suggesting IPMN are segmental or diffuse dilatation of the main PD or focal side-branch dilatation. Sometimes filling defects in the main PD (caused by mucus) can be differentiated from stones by their transient nature when passed with a guidewire. 


\section{Ampullary adenoma}

Before endoscopic resection of an ampullary adenoma, the extent of biliary and pancreatic involvement is evaluated by ERCP. Some authors recommend surgical resection if there is evidence of intraductal extension; other authors still favor endoscopic resection as long as the extension is $<1 \mathrm{~cm}$ because, at this level, the invading tissue can be endoscopically ablated..$^{23}$

\section{Cholangiocarcinoma}

Cholangiocarcinoma is a cancer of the bile ducts. It is rare and highly lethal due to local metastases at presentation. The common risk factor is PSC, which is closely associated with UC. Patients with unresectable cholangiocarcinoma and jaundice should undergo ERCP for stent placement. ${ }^{34}$

\section{Pancreatic malignancy}

Pancreatic cancer is the fourth leading cause of death in the United States. ${ }^{35}$ The pathognomonic finding of pancreatic head cancer on ERCP is the 'double-duct' sign, biliary and pancreatic ductal strictures with proximal dilation. With recent advances in other noninvasive pancreatic imaging modalities, preoperative ERCP does not add further staging information and may result in complications (pancreatitis and perforation) that make surgery more difficult. However, for patients with cholangitis, severe pruritus, or patients who are not fit for surgery, ERCP with biliary stenting should be performed to relieve obstruction. ${ }^{34}$

\section{Indications for EUS}

\section{Esophageal cancer/CRC}

These cancers are staged according to the 'Tumor, Node, and Metastasis' system. Accurate staging is important for therapeutic decision-making and prognostication. Staging with EUS is more accurate than computed tomography (CT) in these types of cancers. ${ }^{13}$

\section{Gastric carcinoma/lymphoma}

Similar to esophageal cancer, EUS is superior to both CT and intraoperative surgical assessment for staging gastric carcinoma and lymphoma. It is also useful in monitoring response to therapy in patients with lymphoma. ${ }^{36}$

\section{Mediastinal adenopathy}

Mediastinal adenopathy may be detected by CT, by chest $\mathrm{X}$-ray, or by the presence of extrinsic compression of the esophagus detected during EGD. Mediastinal adenopathy is caused by both benign processes (sarcoidosis, tuberculosis, and histoplasmosis) and malignant processes (metastatic cancer and lymphoma). EUS-FNA avoids mediastinoscopy or thoracotomy and increases the yield of malignancy. Therefore, EUS-FNA is the preferred procedure for tissue sampling of posterior mediastinal lymph nodes or lesions seen on cross-sectional imaging. ${ }^{37}$

\section{Pancreatic malignancy}

EUS and EUS-FNA are useful adjunctive techniques for the diagnosis and staging of pancreatic malignancies. In patients with unresectable carcinoma, EUS allows patient selection for stent placement and EUS-guided celiac plexus neurolysis. ${ }^{34}$

\section{Pancreatic cystic lesions}

Pancreatic cystic lesions are a frequent incidental finding due to the increased use of high resolution abdominal radiographic imaging. These lesions are classified into either benign or malignant cysts, depending on whether they have the malignant potential. The benign cyst is pseudocyst, whereas the malignant cystic lesions consist of serous cystadenoma, mucinous cystadenoma, IPMN, and solid pseudopapillary neoplasm. ${ }^{33}$ EUS and EUS-guided procedures (EUS-FNA, EUS-guided drainage, and EUSguided celiac plexus blockage) play an important role in diagnosing and managing pancreatic cystic lesions. Some EUS features can differentiate a certain type of cystic lesion from others. EUS-FNA of the cystic lesions provides further information on the etiology of the cysts. The different types of pancreatic cystic lesions include the following:

1. Acute/chronic pseudocyst: A pseudocyst is a collection of pancreatic juice enclosed by a wall of nonepithelialized granulation tissue. Acute pseudocyst, which requires at least 4 weeks to form, arises from acute pancreatitis; chronic pseudocyst develops as a result of chronic pancreatitis. EUS-guided drainage of pseudocysts has similar outcomes to surgical drainage. The indications for drainage are abdominal pain, gastric outlet obstruction, early satiety, weight loss, jaundice, infection, or progressive enlargement, even if asymptomatic. ${ }^{32}$ Long-term pain management in patients with chronic pancreatitis can be difficult. EUS-guided celiac plexus blockage has shown to be more effective, has longer duration, and is less expensive than CT-guided plexus blockage. ${ }^{32}$

2. Serous cystadenoma: Serous cystadenoma originates from centroacinar cells of the pancreatic exocrine system. 
The lesion consists of many small cystic spaces separated by thin septae. Hence, on EUS, it has been described as having a 'honeycomb' or 'sponge-like' appearance. Malignant progression is extremely rare, so surveillance is unnecessary. ${ }^{33}$

3. Mucinous cystadenoma: Mucinous cystadenoma is usually solitary, occurring exclusively in women, and almost always found in the pancreatic body or tail. The presence of ovarian-type stroma is required to diagnosis mucinous cystic neoplasm. Fluid obtained by EUS-FNA is typically viscous and positive for mucin. On EUS, it appears macrocystic and has peripheral calcifications. The rates of malignancy are ranged from $6 \%$ to $27 \%{ }^{38}$ The international consensus guidelines recommend surgical resection in patients who are fit for surgery. ${ }^{39}$ No surveillance is required after resection in patients with benign and noninvasive lesions.

4. IPMN: IPMN is a premalignant lesion involving mucinproducing epithelial cells of the PDs. IPMN affects men and women equally, usually locates in the head of the pancreas, and may be multiple. IPMN is further subdivided into three types: main-duct IPMN, branchduct IPMN, and mixed-duct IPMN. Main-duct IPMN is characterized by dilation of the main duct (more than $1 \mathrm{~cm}$ ), whereas branch-duct IPMN consists of the sidebranch mucinous cyst without main duct involvement. Mixed-duct IPMN has features of both branch-duct and main-duct IPMNs. The prevalence of malignancy in main/mixed-duct IPMN is significantly higher than branch-duct IPMN. According to a pooled analysis, 70\% and $43 \%$ of main/mixed-duct IPMN versus $25 \%$ and $15 \%$ of branch-duct IPMN have carcinoma in situ and invasive carcinomas, respectively. ${ }^{39}$ As a result, the guidelines recommend resection for all patients with main/mixedduct IPMNs who are good surgical candidates and have reasonable life expectancy. ${ }^{39}$ For branch-duct IPMN, resection is suggested for those who have symptoms associated with the cyst (pain or pancreatitis), cystic lesion more than $30 \mathrm{~mm}$, and intramural nodules or cyst fluid cytology suspicious/positive for malignancy. Otherwise, a 'watch and see' approach with frequent surveillance is recommended for 'low-risk' branch-duct IPMN. If the cyst is less than $1 \mathrm{~cm}$ and if imaging studies show stability after 1 year, then the interval of surveillance can be lengthened to 2 years. However, emerging evidence indicates this 'low-risk' entity may be associated with synchronous and/or metachronous pancreatic ductal carcinomas. ${ }^{40}$ As a result, our understanding of the natural history of 'low-risk' branch-duct IPMN is still an enigma. Several EUS findings can distinguish the above three subtypes, which include the main duct and/or branch-duct dilation(s), septation, and mural nodularity (solid components within the cystic lesion). Mural nodularity is a harbinger of malignancy. High cystic carcinoembryonic antigen level obtained by EUS-FNA may also indicate malignancy.

5. Solid pseudopapillary neoplasm: Solid pseudopapillary neoplasm is a rare pancreatic cyst lesion with low malignant potential. It is commonly seen in young women $(<35$ years). The characteristics on EUS are well-demarcated, echo-poor, and solid-appearing mass. ${ }^{33}$ Fluid analysis by EUS-FNA can diagnose $75 \%$ of cases. ${ }^{38}$ Because of the indolent nature of this lesion, even with metastases, surgery is still recommended.

\section{Submucosal tumors}

Submucosal tumors are divided into two groups. The more common group is referred to as gastrointestinal stromal tumors, and the less common group includes lipomas, schwannomas, hemangiomas, leiomyomas, and their malignant counterparts (leiomyosarcoma). The diagnosis of these tumors is difficult by standard imaging. EUS can suggest the nature of a submucosal tumor by determining the layer of origin and echotexture of the lesion. Tumor size, an irregular border, echogenic foci, and cystic spaces are independently associated with malignancy. ${ }^{36}$

\section{Ampullary adenoma/NDA}

For ampullary adenoma, EUS and intraductal EUS (a small EUS probe passing inside the pancreatobiliary systems) allow us to evaluate periampullary lymph nodes, to select patients for endoscopic versus surgical resection, and to guide surgical therapy. ${ }^{23}$ For NDA, EUS allows us to determine the relationship of the polyp to the pancreaticobiliary tree when side-viewing examination is uncertain. In addition, EUS can also determine whether endoscopic removal is feasible when biopsy shows HGD. ${ }^{23}$

\section{Ampullary carcinoma}

Ampullary carcinoma is a relatively rare cancer that accounts for approximately $0.2 \%$ of GI malignancies. ${ }^{41}$ It is suspected when a patient presents with obstructive jaundice, and abdominal imaging studies show pancreatic and biliary ductal dilatation. EUS allows not only more accurate diagnosis and staging of these lesions than CT, but it also permits tissue biopsies and FNA sampling. ${ }^{34}$ In addition, 
EUS selects appropriate patients for undergoing local resection instead of pancreaticoduodenectomy (Whipple procedure).

\section{IBD}

For patients with CD, EUS can accurately assess perianal fistula and abscess. EUS is also useful in monitoring medical and surgical therapy for $\mathrm{CD}$ perianal fistulae. ${ }^{24}$

\section{Indications for WCE/DBE Obscure GI bleed}

Two modalities have complementary roles and are not mutually exclusive. The most common indication of WCE is for the evaluation of obscure GI bleeding (unknown bleeding origin after initial EGD and colonoscopy). A cost-analysis study showed that the initial DBE approach is more costeffective. ${ }^{42}$ However, because DBE is potentially associated with serious complications, such as pancreatitis and bowel perforation, the authors advocate using CE-directed DBE approach because this would reduce complications and decreased utilization of endoscopic resources. ${ }^{42}$

\section{Other indications}

Other indications for WCE include CD, ulcers, polyps, foreign bodies, infiltrative diseases, and imaging abnormalities. ${ }^{6}$ It should be emphasized that in patients with $\mathrm{CD}$ and suspected strictures, WCE is contraindicated.

\section{Indications for colonoscopy Acute colonic pseudo-obstruction}

Acute colonic pseudo-obstruction (ACPO) is characterized by massive colonic dilation in the absence of mechanical obstruction. The symptoms include nausea, vomiting, abdominal pain, constipation, and paradoxically diarrhea. Spontaneous perforation occurs in $3 \%-15 \%$ of patients with a mortality rate of $50 \%$ or higher. Endoscopic decompression should be performed if conservative therapy fails or if neostigmine is contraindicated. ${ }^{43}$

\section{Chronic diarrhea}

Lower endoscopy can play a pivotal role in the evaluation of patients with chronic diarrhea. Endoscopy with colonic biopsy is helpful in ruling out IBD, ischemic colitis, collagenous and microscopic colitis, and neoplastic disease. When patients with human immunodeficiency virus and chronic diarrhea have negative basic stool test, lower endoscopy with biopsy is indicated to rule out infectious causes. Mucosal biopsies are the 'gold standard' to establish graft-versus-host-disease after bone marrow transplantation and to rule out other infections and pathologies. Upper GI evaluation may ensue when lower GI workup for chronic diarrhea is negative. ${ }^{44}$

\section{Ampullary adenoma/NDA}

Studies indicate that patients with sporadic ampullary adenomas or duodenal adenomas are at greater risk for CRC. ${ }^{23}$ Screening colonoscopy should be offered to patients with these polyps.

\section{IBD}

Mucosal biopsy during colonoscopy with ileoscopy is invaluable in the evaluation of IBD and the differentiation of UC from $\mathrm{CD}$. Endoscopy along with other diagnostic modalities can distinguish CD from UC in $\geq 85 \%$ of patients. ${ }^{24}$ Surveillance colonoscopy every $1-2$ years starting $8-10$ years after disease onset is recommended for patients with $\mathrm{CD}$ and $\mathrm{UC}$. Biopsy specimens are obtained in all four quadrants every $10 \mathrm{~cm}$ from the cecum to the rectum. Colectomy should be performed when there is the presence of HGD or multifocal LGD in a flat mucosa. Colonic strictures in patients with $\mathrm{UC}$ are considered malignant until proven otherwise. If a stricture is unable to be assessed thoroughly, colectomy is indicated. ${ }^{24}$ Chronic obstruction due to fibrotic strictures can be managed by endoscopic balloon dilation. For patients with CD undergoing partial colectomy or partial ileocolectomy, recurrence rates range from $70 \%$ to $90 \%$ after 1 year of surgery. ${ }^{24}$ Therefore, colonoscopy is recommended at 6-12 months after surgery to identify patients who may benefit from medical therapy.

\section{Colorectal screening and surveillance}

CRC is the second leading cause of cancer-related deaths in the United States. Therefore, it is important for primary care physicians to understand the role of CRC screening and surveillance. The following recommendations for early detection of CRC and adenomatous polyps in average-risk adults (aged 50 years and older) are based on the most recent published guideline by the ACS..$^{45}$ For CRC screening and surveillance in high-risk patients (family or personal history of CRC, IBD, or FAP/HNPCC), please refer to the actual guidelines. ${ }^{45}$ Screening tests are divided into two categories: fecal tests (guaiac fecal occult blood test (gFOBT), fecal immunochemical test (FIT), and stool-based DNA (sDNA)) and structural exams (FS, colonoscopy, double contrast barium enema (DCBE), and computerized topography colonography (CTC)). The appropriate test to be used 
depends on the individual's preference and local resources. Patients should understand that positive findings with less invasive tests require follow-up colonoscopy.

1. gFOBT: Annual screening with gFOBT has high sensitivity for detecting CRC in average-risk adults aged 50 years and older. Two to three stool samples from each of three consecutive bowel movements at home are collected. Guaiac testing following digital rectal exam in the office and rehydrated specimen (adding distilled water to the dry sample) are not recommended. Patients are instructed to avoid aspirin or NSAIDS 7 days prior to testing, unless they are on a cardioprotective regimen. Because the gFOBT is based on the detection of peroxidase, certain foods (citrus fruits, turnip, horseradish, and red meats) should also be avoided 3 days before testing. If any test is positive, a follow-up colonoscopy is recommended. Primary practitioners should emphasize the importance of annual screening in order to achieve the fullest potential of this test.

2. FIT: FIT detects human hemoglobin, which eliminates the need of a restricted diet before testing as with gFOBT. In addition, FIT requires fewer specimens (2 versus 3 ) and simpler sampling procedure (brush versus spatula method) when compared with guaiac-based testing. Currently, there are no data to support the superiority of FIT over gFOBT. However, the likelihood of test completion appears to be greater with FIT due to its simplicity.

3. sDNA: Adenoma and CRC that contained altered DNA are shed and passed in feces. The abnormal DNA is isolated and detected by a multiple-marker panel. Because sDNA is an evolving technology, the screening interval between negative tests remains to be determined.

4. FS: FS will be discussed later.

5. Colonoscopy: The evidence to support screening colonoscopy is substantial. At present, screening colonoscopy every 10 years is recommended.

6. DCBE: As the name implies, the entire colon is distended with air and barium via a rectal catheter. Multiple radiographs are acquired during a 20-40-min procedure. Because no sedatives are given, patients can return to work after the examination. The acceptability of DCBE may be limited by requiring a $24-\mathrm{h}$ bowel preparation, and some patients may experience mild to moderate discomfort during and after the procedure. In addition, DCBE has lower sensitivity than colonoscopy and provides no opportunity for biopsy. A follow-up colonoscopy is required for any polyps greater than $6 \mathrm{~mm}$ on DCBE. A 5-year interval screening is recommended.
7. CTC: Thin CT images of the large intestines are acquired in 10-min procedure. Bowel preparation is similar to colonoscopy. The colon is distended by room air or carbon dioxide via a catheter in the rectum. No sedation is required during the procedure. If the CTC shows any polyps larger, equal to $6 \mathrm{~mm}$ or greater, colonoscopy should be offered. CTC is appropriate for those patients who decline colonoscopy or when colonoscopy is contraindicated. Screening interval is 5 years for average-risk patients at age of 50 .

8. $C R C$ : Colonoscopy allows for multiple endoscopic biopsies necessary to confirm CRC. Malignant obstruction can be managed effectively for palliation, or as a bridge to surgery with self-expandable metal stent or laser therapy. HGD and malignant pedunculated polyps with favorable histologic features can be adequately treated with endoscopic resection. Surveillance should consist of a follow-up endoscopy within 3-6 months after resection. ${ }^{46}$

\section{Constipation}

Chronic constipation is associated with an increased risk of colon cancer. ${ }^{47}$ For this reason, patients with constipation who are above 50 years and have not had a colon cancer screening should have a colonoscopy. ${ }^{47}$ Patients and physicians should be aware that Medicare (health insurance program for the elderly administered by the US government) might not reimburse for colonoscopy if the sole indication is constipation.

\section{Lower Gl Bleeding}

Lower GI bleeding (LGIB) may present as occult fecal blood, iron deficiency anemia, melena, intermittent hematochezia, or acute bleeding. Colonoscopy is effective in the diagnosis and treatment of LGIB. If a bleeding source is not identified by colonoscopy, EGD should be performed to rule out upper GI bleeding. ${ }^{48}$

\section{Indications for FS Colorectal screening}

Patients with distal adenomas have an increased risk of advanced proximal neoplasia. If adenomas are found at FS, colonoscopy should be performed for complete examination of the colon. For surveillance of average-risk patients, either FS alone or FS with annual gFOBT or FIT is recommended every 5 years. $^{45}$

\section{Other indications}

Because of low malignant risk in younger patients ( $<50$ years) with suspected distal colonic disease, FS should be considered 
instead of colonoscopy. In patients with IBD, FS is preferred when colonoscopy is considered high risk (fulminant colitis). In addition, when there is an exacerbation in patients with UC, FS is useful in evaluating superimposed infections (cytomegalovirus, Clostridium difficile) or condition (ischemic colitis). ${ }^{24}$

\section{Conclusion}

In the rapidly changing field of endoscopy as well as increasing referrals to OAE centers by nongastroenterologists, primary care practitioners should have an updated understanding of new endoscopic technologies and its indications in order to save resources, to provide optimal care with the least risk, and to facilitate effective communication between different specialties.

\section{Disclosure}

The authors report no conflicts of interest in this work.

\section{References}

1. Charles RJ, Chak A, Cooper GS, et al. Use of open access in GI endoscopy at an academic medical center. Gastrointest Endosc. 1999; 50:480-485.

2. Waring JP, Baron TH, Hirota WK, et al. Guidelines for conscious sedation and monitoring during gastrointestinal endoscopy. Gastrointest Endosc. 2003;58:317-322.

3. Faigel DO, Eisen GM, Baron TH, et al. Preparation of patients for GI endoscopy. Gastrointest Endosc. 2003;57:446-450.

4. Wexner SD, Beck DE, Baron TH, et al. A consensus document on bowel preparation before colonoscopy: prepared by a task force from the American Society of Colon and Rectal Surgeons (ASCRS), the American Society for Gastrointestinal Endoscopy (ASGE), and the Society of American Gastrointestinal and Endoscopic Surgeons (SAGES). Gastrointest Endosc. 2006;63:894-908.

5. Mishkin DS, Chuttani R, Croffie J, et al. ASGE Technology Status Evaluation Report: wireless capsule endoscopy. Gastrointest Endosc. 2006;63:539-545.

6. DiSario JA, Petersen BT, Tierney WM, et al. Technology status evaluation report: enteroscopes. Gastrointest Endosc. 2007;66:872-880.

7. Mensink PB, Haringsma J, Kucharzik T, et al. Complications of double balloon enteroscopy: a multicenter survey. Endoscopy. 2007;39(7): 613-615.

8. Möschler O, May AD, Müller MK, Ell C; DBE-Studiengruppe Deutschland. Complications in double-balloon-enteroscopy: results of the German DBE register. Z Gastroenterol. 2008;46(3):266-270.

9. Richter JE. Achalasia. In: Castell DO, Richter JE, editors. The esophagus. 4th ed. Philadelphia: Lippincott Williams \& Wilkins; 2004:221-261.

10. Sharma P, Sampliner RE. Barrett's esophagus. In: Castell DO, Richter JE, editors. The esophagus. 4th ed. Philadelphia: Lippincott Williams \& Wilkins; 2004:497-506.

11. Hirota WK, Zuckerman MJ, Adler DG, et al. ASGE guideline: the role of endoscopy in the surveillance of premalignant conditions of the upper GI tract. Gastrointest Endosc. 2006;63:570-580.

12. Tytgat GN. Benign and malignant tumors of the esophagus. In: Sivak MV, editor. Gastroenterologic Endoscopy. 2nd ed. Philadelphia: W.B. Saunders Company; 2000:501-520.

13. Jacobson BC, Hirota W, Baron TH, et al. ASGE guideline. The role of endoscopy in the assessment and treatment of esophageal cancer. Gastrointest Endosc. 2003;57:817-822.
14. Johnston MH, Wong R. Esophageal strictures. In: Castell DO, Richter JE, editors. The esophagus. 4th ed. Philadelphia: Lippincott Williams \& Wilkins; 2004:507-517.

15. Egan JV, Baron TH, Adler DG, et al. Esophageal dilation. Gastrointest Endosc. 2006;63:755-760.

16. Qureshi W, Adler DG, Davila R, et al. The role of endoscopy in the management of variceal hemorrhage, updated Jul 2005. Gastrointest Endosc. 2005;62:651-655.

17. Eisen GM, Baron TH, Dominitz JA, et al. Guideline for the management of ingested foreign bodies. Gastrointest Endosc. 2002;55:802-806

18. Ikenberry SO, Harrison ME, Lichtenstein D, et al. The role of endoscopy in dyspepsia. Gastrointest Endosc. 2007;66(6):1071-1075.

19. Dooley CP, Larson AW, Stace NH, et al. Double-contrast barium meal and upper gastrointestinal endoscopy. A comparative study. Ann Intern Med. 1984;101(4):538-545.

20. ASGE guideline. The role of endoscopy in the management of GERD. Gastrointest Endosc. 1999;49:834-835.

21. Garcia M, Jemal A, Ward EM, et al. Global cancer facts and figures 2007. American Cancer Society. Available from: http://www. cancer.org/downloads/STT/Global_Facts_and_Figures_2007_rev2. pdf. Accessed 2009 Sep 19.

22. ASGE guideline. The role of endoscopy in the management of the patient with peptic ulcer disease. Guidelines for clinical application. Gastrointest Endosc. 1988;34 Suppl 3:21S-22S.

23. Adler DG, Qureshi W, Davila R, et al. The role of endoscopy in ampullary and duodenal adenomas. Gastrointest Endosc. 2006;64(6):849-854.

24. Leighton JA, Shen B, Baron TH, et al. ASGE guideline: endoscopy in the diagnosis and treatment of inflammatory bowel disease. Gastrointest Endosc. 2006;63(4):558-565.

25. Eisen GM, Baron TH, Faigel DO, et al. ASGE guideline. Role of endoscopy in enteral feeding. Gastrointest Endosc. 2002;55:794-797.

26. Adler DG, Leighton JA, Davila RE, et al. ASGE guideline: the role of endoscopy in acute non-variceal upper-GI hemorrhage. Gastrointest Endosc. 2004;60:497-504.

27. Zaman A, Katon RM. Push enteroscopy for obscure gastrointestinal bleeding yields a high incidence of proximal lesions within reach of a standard endoscope. Gastrointest Endosc. 1998;47(5):372-376.

28. An JY, Hayman LL, Park YS, et al. Web-based weight management programs for children and adolescents: a systematic review of randomized controlled trial studies. ANS Adv Nurs Sci. 2009;32(3):222-240.

29. Anderson MA, Gan SI, Fanelli RD, et al. Role of endoscopy in the bariatric surgery patient. Gastrointest Endosc. 2008;68(1):1-10.

30. Adler DG, Baron TH, Davila RE, et al. ASGE guideline: the role of ERCP in disease of the biliary tract and the pancreas. Gastrointest Endosc. 2005;62:1-8.

31. Car-Locke DL. Therapeutic role of ERCP in the management of suspected common bile duct stones. Gastrointest Endosc. 2002;56 Suppl 6: S170-S174.

32. Adler DG, Lichtenstein D, Baron TH, et al. The role of endoscopy in patients with chronic pancreatitis. Gastrointest Endosc. 2006;63: 933-937.

33. Jacobson BC, Baron TH, Adler DG, et al. ASGE guideline. The role of endoscopy in the diagnosis and the management of cystic lesions and inflammatory fluid collections of the pancreas. Gastrointest Endosc. 2005;61(3):363-370.

34. Baron TH, Mallery JS, Hirota WK, et al. The role of endoscopy in the evaluation and treatment of patients with pancreaticobiliary malignancy. Gastrointest Endosc. 2003;58:643-649.

35. Parker SL, Tong T, Bolden S, Wingo PA. Cancer statistics, 1997. CA Cancer J Clin. 1997;47(1):5-27.

36. Eisen GM, Chutkan R, Goldstein JL, et al. ASGE guideline. Role of endoscopic ultrasonography. Gastrointest Endosc. 2000;52:852-859.

37. Jacobson BC, Hirota WK, Goldstein JL, et al. The role of EUS for evaluation of mediastinal adenopathy. Gastrointest Endosc. 2003;58: 819-821. 
38. Fasanella KE, McGrath K. Cystic lesions and intraductal neoplasms of the pancreas. Best Pract Res Clin Gastroenterol. 2009;23(1): 35-48.

39. Tanaka M, Chari S, Adsay V, et al. International consensus guidelines for management of intraductal papillary mucinous neoplasms and mucinous cystic neoplasms of the pancreas. Pancreatology. 2006;6: 17-32.

40. Nguyen VX, Decker GA, Das A, et al. The natural history of a branch duct intraductal papillary mucinous neoplasm in a patient with Lady Windermere syndrome: a case report. JOP. 2010;11(3):249-254.

41. Albores-Saavedra J, SchwartzAM, Batich K, et al. Cancers of the ampulla of vater: demographics, morphology, and survival based on 5,625 cases from the SEER program. J Surg Oncol. 2009;100(7):598-605.

42. Gerson L, Kamal A. Cost-effectiveness analysis of management strategies for obscure GI bleeding. Gastrointest Endosc. 2008;68(5): 920-936.

43. Eisen GM, Baron TH, Dominitz JA, et al. Acute colonic pseudoobstruction. Gastrointest Endosc. 2002;56:789-792.
44. Eisen GM, Dominitz JA, Faigel DO, et al.ASGE guideline. Use of endoscopy in diarrheal illnesses. Gastrointest Endosc. 2001;54: 821-823.

45. Levin B, Lieberman DA, McFarland B, et al. Screening and surveillance for the early detection of colorectal cancer and adenomatous polyps, 2008: a joint guideline from the American Cancer Society, the US MultiSociety Task Force on colorectal cancer, and the American College of Radiology. CA Cancer J Clin. 2008;58:130-160.

46. Davila RE, Rajan E, Adler D, et al. ASGE guideline: the role of endoscopy in the diagnosis, staging, and management of colorectal cancer. Gastrointest Endosc. 2005;61:1-7.

47. Qureshi W, Adler DG, Davila R, et al. ASGE guideline: guideline on the use of endoscopy in the management of constipation. Gastrointest Endosc. 2005;62:199-201.

48. Davila RE, Rajan E, Adler DG, et al. ASGE guideline: the role of endoscopy in the patient with lower-GI bleeding. Gastrointest Endosc. $2005 ; 62: 656-660$
International Journal of General Medicine

\section{Publish your work in this journal}

The International Journal of General Medicine is an international, peer-reviewed open-access journal that focuses on general and internal medicine, pathogenesis, epidemiology, diagnosis, monitoring and treatment protocols. The journal is characterized by the rapid reporting of reviews, original research and clinical studies across all disease areas.

\section{Dovepress}

A key focus is the elucidation of disease processes and management protocols resulting in improved outcomes for the patient.The manuscript management system is completely online and includes a very quick and fair peer-review system. Visit http://www.dovepress.com/ testimonials.php to read real quotes from published authors.

Submit your manuscript here: http://www.dovepress.com/international-journal-of-general-medicine-journal 